\title{
A FILM MODEL FOR HEAT AND MASS TRANSFER WITH FOG FORMATION
}

\author{
H. J. H. BROUWERS ${ }^{\dagger}$ \\ Akzo Research Laboratories Arnhem, Fibers Division, Department of Mechanical Engineering, \\ Velperweg 76, 6824 BM Arnhem, The Netherlands
}

(Received 11 March 1991; accepted for publication 9 December 1991)

\begin{abstract}
An analysis is presented of a binary film with fog formation and a negligible induced velocity (traditionally referred to as "Stefan-Nusselt flow"). The governing equations of energy and diffusion, coupled with the saturation condition, are solved and analytical correction factors are derived. Subsequently, the "negligible induced velocity" (NIV) fog film model is applied to channel flow, yielding analytical expressions for the variation of bulk vapour mass fraction, bulk temperature, and the possible creation of bulk fog. Multiplying the NIV correction factor for fog only by the classical film model correction factors for induced velocity, reveals that the product corresponds to the film model correction factors for the combined effects of fog and induced velocity. Furthermore, a thorough comparison with theoretical and experimental results of foregoing two-dimensional studies, concerning fog formation in the presence of free and forced convection, confirms the accuracy of the present fog film model.
\end{abstract}

\section{INTRODLCTION}

The classical film model correction factors for the effect of suction and injection were derived and applied by Brouwers and Chesters (1992) and extended to include the formation of fog by Brouwers (1992). This new film model provides correction factors which account for the effect of both an induced velocity and fog formation. The calculated results illustrated that, for small vapour mass fractions, implying in turn small induced velocities, the effect of fog formation on heat and mass transfer is greatest. As this interaction between fog-related heat and mass transfer is extremely interesting and the governing equations are significantly simplified without induced velocity, in this paper the limiting case is analysed in which fog is formed in the film but the vapour mass fraction is small. Moreover, Fig. 1 reveals that the

'Present address: Department of Civil Engineering, Twente University, P.O. Box 217, 7500 AE Enschede, The Netherlands. study of this physical situation completes the film model analyses for transport phenomena in the presence of fog formation and/or induced velocity.

Formation of fog in dilute, vapour non-condensable mixtures has been the subject of numerous investigations in the past. In the introduction of Brouwers (1992), the studies based on the "critical supersaturation model" (CSM) and on the saturation condition have already been discussed. Studies of fog formation in dilute one-dimensional systems, in combination with the saturation condition, have been carried out by Hills and Szekely $(1964,1969)$ and Toor $(1971 \mathrm{a}, \mathrm{b})$. The former investigators proceeded from saturated bulk properties and an entirely saturated film. The latter author determined the existence of a saturated region and its boundary with the superheated region, employing the tangency condition. All the theories presented, however, were limited to cases where $L e=1$ (and $\delta_{i}=\delta_{c}$ ) and not brought within the scope of a general fog film model which can be applied to convective heat and mass transfer.

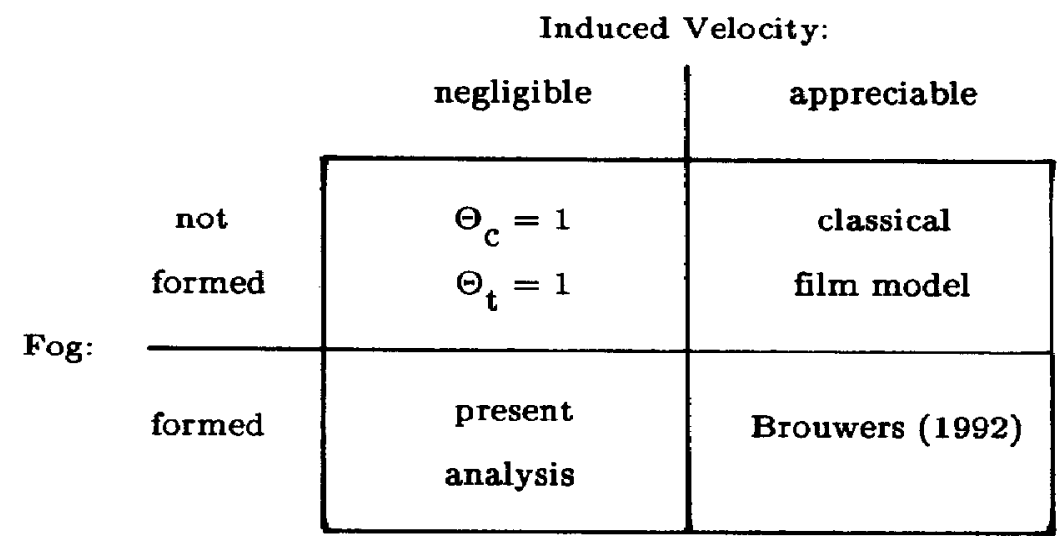

Fig. 1. Review of film model analyses including and excluding induced velocity and fog formation. 
Here then, a film of a binary mixture with any $L e$ value will be considered but without induced velocity, this physical simplification being permissible when the vapour mass fraction is sufficiently small. The error introduced by omitting this velocity will be assessed thoroughly by means of an asymptotic analysis.

The possible existence and magnitude of a fog layer in the film will be determined and explained graphically. The simplified governing equations of heat and mass transfer in the film are treated analytically, and compact and useful correction factors are derived. These correction factors for heat and mass transfer give a clear insight into the influence of the diverse parameters on fog formation.

The film model approach to fog formation is then applied to closed-channel flow and analytical expressions for the bulk vapour mass fraction and bulk temperature variation in the channel derived. Since attention is focused only on the interaction between the energy and diffusion equations when fog is formed (and not on the effect and presence of the induced velocity in these equations), the analysis presented here will not yield a correction for the friction coefficient, as this is affected only by suction or injection.

Furthermore, it will be demonstrated that the derived fog correction factors can be adapted easily to heat and mass transfer in the presence of suction or injection. In this way, a compound fog film model is derived which is based on relatively simple equations, and can be applied to heat and mass transfer in the presence of both fog formation and an induced velo-
The present film analysis is based on:

- the saturation condition [i.e. $c=F(T)]$, and - no induced velocity (NIV).

The so-called saturation condition has been utilized frequently in the past. The saturation condition holds in general when sufficient particles are present in the mixture which can serve as nuclei for condensation. According to Steinmeyer (1972) these conditions are indeed often fulfilled in practical situations. The level of supersaturation, which thermodynamically must always be non-zero, can then be regarded as negligibly small. Here, the saturation condition is employed in view of its convenience and accuracy to most practical situations. In the analysis of the fog layer, the droplets created (and transported, e.g. by thermophoresis) are not explicitly considered and the physical properties in the superheated and saturated mixture are therefore assumed to be identical. This approach is quite acceptable since the fraction of droplets in mixtures is usually very small.

The error introduced by not taking the induced velocity into account is assessed with the help of an asymptotic analysis of the relation between vapour mass fraction and temperature, $G(T)$, for small vapour mass fractions. Since the temperature and vapour mass fraction profiles tend to a linear dependence on $y$ for small vapour mass fractions, the relation between $c$ and $T$ will be linear as well. The relation between $c$ and $T$ in a film with induced velocity is represented by

$\delta_{c}<\delta_{t}: \begin{cases}c=G(t)=1+\left(c_{i}-1\right)\left\{\frac{T-T_{i}}{T_{b}-T_{i}}\left[e^{\left(\delta_{t} / L e_{v} \delta_{c}\right) \ln \left(1-c_{b} / 1-c_{i}\right)}-1\right]+1\right\}^{L e_{\nu}} & {\left[T_{i} \leqslant T \leqslant T\left(y=\delta_{c}\right)\right]} \\ c=c_{b} & {\left[T\left(y=\delta_{c}\right) \leqslant T \leqslant T_{b}\right]}\end{cases}$

$\delta_{c}=\delta_{t}: \quad c=G(t)=1+\left(c_{i}-1\right)\left\{\frac{T-T_{i}}{T_{b}-T_{i}}\left[e^{\left(1 / L e_{v}\right) \ln \left(1-c_{b} / 1-c_{i}\right)}-1\right]+1\right\}^{L e_{v}} \quad\left(T_{i} \leqslant T \leqslant T_{b}\right)$

$\delta_{c}>\delta_{t}:\left\{\begin{array}{l}c=G(t)=1+\left(c_{i}-1\right)\left\{\frac{T-T_{i}}{T_{b}-T_{i}}\left[e^{\left(\delta_{t} / L e_{v} \delta_{e}\right) \ln \left(1-c_{b} / 1-c_{i}\right)}-1\right]+1\right\}^{L e_{v}} \\ c \text { is not a function of } T \text { for } \delta_{t}<y \leqslant \delta_{c} .\end{array}\right.$

city. Finally, the fog film model is compared extensively with theoretical and experimental results of two-dimensional free and forced convective heat and mass transfer analyses, performed by previous investigators.

\section{ASYMPTOTIC ANALYSIS OF THE FILM FOR A NEGLIGIBLE INDUCED VELOCITY (NIV)}

By considering a binary stagnant film next to a wall (e.g. denoting the surface of a liquid film) Brouwers and Chesters (1992) derived expressions for the temperature, $T$, and vapour mass fraction, $c$, as a function of the coordinate, $y$, perpendicular to the wall. Their analysis reckoned with the effect of the induced velocity, but not with the formation of fog in the film. [see Brouwers and Chesters (1992).] $G(T)$ is furthermore linear if $L e_{v}$ is equal to unity, regardless of the magnitude of $c$. $G(T)$ therefore tends to a linear function of $T$ if both $\varepsilon_{1}$ and $\varepsilon_{2}$ are close to zero, where

$$
\varepsilon_{1}=\frac{c_{b}(x=0)-c_{i}}{1-c_{i}}
$$

and

$$
\varepsilon_{2}=L e_{v}-1 \text {. }
$$

In eq. (2), the entrance vapour mass fraction, $c_{b}(x=0)$, figures since this is the most extreme condition. In a channel, the local bulk vapour mass fraction is always situated between the entry value and the vapour fraction at the wall, $c_{i} . G(T)$ can now be 
asymptotically expanded for small $\varepsilon_{1}$ and $\varepsilon_{2}$ as fol-

lows:

$\delta_{c}<\delta_{t}: \begin{cases}c=G(T)=c_{i}+\left(c_{b}-c_{i}\right) \frac{\delta_{t}}{\delta_{c}}\left(\frac{T-T_{i}}{T_{b}-T_{i}}\right)\left[1+O\left(\varepsilon_{1} \varepsilon_{2}\right)\right] & {\left[T_{t} \leqslant T \leqslant T\left(y=\delta_{c}\right)\right]} \\ c=c_{b} & {\left[T\left(y=\delta_{c}\right) \leqslant T \leqslant T_{b}\right]}\end{cases}$

$\delta_{c}=\delta_{t}: c=G(T)=c_{i}+\left(c_{b}-c_{i}\right)\left(\frac{T-T_{i}}{T_{b}-T_{i}}\right)\left[1+O\left(\varepsilon_{1} \varepsilon_{2}\right)\right] \quad\left(T_{i} \leqslant T \leqslant T_{b}\right)$

$\delta_{c}>\delta_{i}:\left\{\begin{array}{l}c=G(T)=c_{i}+\left(c_{b}-c_{i}\right) \frac{\delta_{t}}{\delta_{c}}\left(\frac{T-T_{i}}{T_{b}-T_{i}}\right)\left[1+O\left(\varepsilon_{1} \varepsilon_{2}\right)\right] \quad\left(T_{i} \leqslant T \leqslant T_{b}\right) \\ c \text { is not a function of } T \text { for } \delta_{t}<y \leqslant \delta_{c} .\end{array}\right.$

The underlying theory of the applied techniques can be found in the standard work of van Dyke (1975).

Equation (4) indicates the small influence of the induced velocity for small $\varepsilon_{1}$. Accordingly, in this paper the induced velocity is not considered and the first-order approximation of $G(T)$, which corresponds to eq. (4) (with $\varepsilon_{1}=0$ substituted), for small $\varepsilon_{1}$ will be employed from now on.

To verify whether fog is formed, the slope conditions of Brouwers (1992) are used. For condensation it reads:

$$
\left.\frac{\mathrm{d} F}{\mathrm{~d} T}\right|_{T_{t}}<\left.\frac{\mathrm{d} G}{\mathrm{~d} T}\right|_{T_{i}}
$$

superheated region, defining

$$
\varepsilon_{3}=\frac{c_{b}-c_{a}}{1-c_{a}}
$$

the zero-order approximation of the temperature distribution for small $\left|\varepsilon_{3}\right|$ reads [see Brouwers (1992)]

$$
T(y)=\frac{T_{b}\left(y-\delta_{a}\right)-T_{a}\left(y-\delta_{t}\right)}{\left(\delta_{t}-\delta_{a}\right)} \quad\left(\delta_{a} \leqslant y \leqslant \delta_{r}\right) .
$$

The first-order approximation of the vapour fraction for small $\left|\varepsilon_{3}\right|$ in the superheated region reads

$$
c(y)=\frac{c_{b}\left(y-\delta_{a}\right)-c_{a}\left(y-\delta_{c}\right)}{\left(\delta_{c}-\delta_{a}\right)} \quad\left(\delta_{a} \leqslant y \leqslant \delta_{c}\right)
$$

Eliminating $y$ from eqs (8) and (9) results in

$$
\begin{aligned}
& \delta_{c}<\delta_{t}: \begin{cases}c=G(T)=\frac{T-T_{a}}{T_{b}-T_{a}} \frac{\delta_{t}-\delta_{a}}{\delta_{c}-\delta_{a}}\left(c_{b}-c_{a}\right)+c_{a} & {\left[T_{a} \leqslant T \leqslant T\left(y=\delta_{c}\right)\right]} \\
c=c_{b} & {\left[T\left(y=\delta_{c}\right) \leqslant T \leqslant T_{b}\right]}\end{cases} \\
& \delta_{c}=\delta_{t}: \quad c=G(T)=\frac{T-T_{a}}{T_{b}-T_{a}\left(c_{b}-c_{a}\right)+c_{a} \quad\left(T_{a} \leqslant T \leqslant T_{b}\right)} \\
& \delta_{c}>\delta_{t}: \begin{cases}c=G(T)=\frac{T-T_{a}}{T_{b}-T_{a}} \frac{\delta_{t}-\delta_{a}}{\delta_{c}-\delta_{a}}\left(c_{b}-c_{a}\right)+c_{a} & \left(T_{a} \leqslant T \leqslant T_{b}\right) \\
c \text { is not a function of } T \text { for } \delta_{t}<y \leqslant \delta_{c} .\end{cases}
\end{aligned}
$$

and for evaporation

$$
\left.\frac{\mathrm{d} F}{\mathrm{~d} T}\right|_{T_{t}}>\left.\frac{\mathrm{d} G}{\mathrm{~d} T}\right|_{T_{t}}
$$

The possible intersection of the saturation line, implying the formation of fog, is detected with eqs (5) and (6), combined with eq. (4), with $\varepsilon_{1}$ set equal to zero.

When these equations predict formation of fog in the film (see Fig. 2), a superheated and a saturated region are distinguished. In $0 \leqslant y \leqslant \delta_{a}$, the film is saturated, and in $\delta_{a} \leqslant y \leqslant \delta_{c}$ or $\delta_{t}$ the film is superheated. The fog film thickness, $\delta_{a}$, is yet to be determined. At the boundary of the superheated and saturated regions the temperature and vapour fraction are defined as $T_{a}$ and $c_{a}\left[=F\left(T_{a}\right)\right]$, respectively. In the

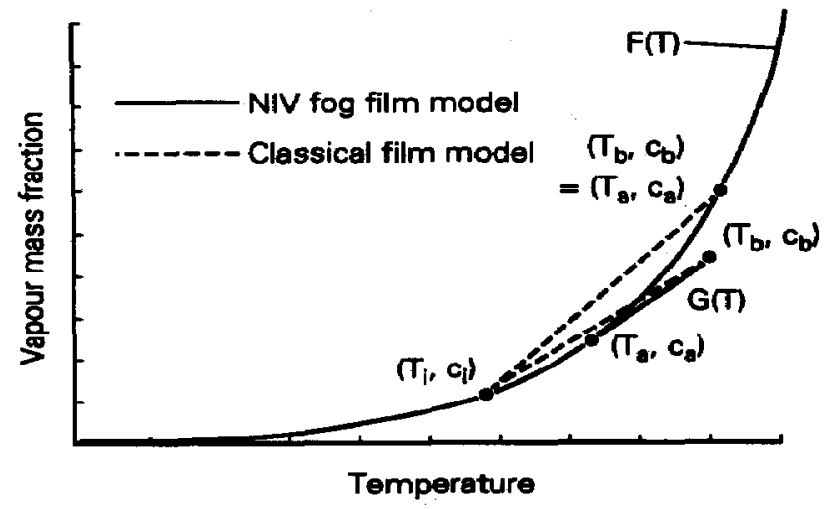

Fig. 2. Determination of the boundary $\left(T_{a}, c_{a}\right)$ of the superheated and saturated regions for two $\left(T_{b}, c_{b}\right), \delta_{t}=\delta_{c}$. 
Relation (10) is linear since both $c$ and $T$ depend linearly on $y$ in the superheated region. For $\delta_{a}=0$ (i.e. $T_{a}=T_{i}$ and $c_{a}=c_{i}$ ), eqs (8) and (9) correspond to the temperature and vapour mass fraction profiles of a film without fog formation and without induced velocity, which are used as reference. In that case, eq. (10) then corresponds to eq. (4) with $\varepsilon_{1}=0$.

As no solution in closed form for the governing equations of the fog layer has been derived yet, it is not possible to derive approximate expressions for small $c$ from them. Accordingly, the basic equations are first linearized and then solved. The linearized diffusion equation in the fog layer (in the limit of small c) reads

$$
\rho \in \frac{\mathrm{d}^{2} c}{\mathrm{~d} y^{2}}=K
$$

with the associated boundary conditions

$$
\begin{gathered}
c(y=0)=c_{i} \\
c\left(y=\delta_{a}\right)=c_{a} .
\end{gathered}
$$

The energy equation in the fog layer, without induced velocity reads

$$
k \frac{\mathrm{d}^{2} T}{\mathrm{~d} y^{2}}=-H_{1 \mathrm{at}} K
$$

with the appropriate boundary conditions

$$
\begin{gathered}
T(y=0)=T_{i} \\
T\left(y=\delta_{a}\right)=T_{a} .
\end{gathered}
$$

In the fog layer, $0 \leqslant y \leqslant \delta_{a}$, and on the borders $y=0$ and $y=\delta_{a}$, the vapour fraction is related to the temperature by the saturation line, $F(T)$. The amount of fog formed, $K$, is eliminated from eqs (11) and (13), the resulting equation is integrated twice with respect to $y$, and boundary conditions (12) and (14) are applied, yielding

$$
\begin{aligned}
\frac{H_{\text {lat }}}{c_{p}} c(y) & +\operatorname{Le} T(y)=\frac{H_{\text {lat }}}{c_{p}} \frac{y\left(c_{a}-c_{i}\right)+c_{i} \delta_{a}}{\delta_{a}} \\
& +\operatorname{Le} \frac{y\left(T_{a}-T_{i}\right)+T_{i} \delta_{a}}{\delta_{a}} \quad\left(0 \leqslant y \leqslant \delta_{a}\right) .
\end{aligned}
$$

This equation is an implicit solution of $T$ as a function of $y$, because $c$ is prescribed by $F(T)$ in the range, $T_{i} \leqslant T \leqslant T_{a}$, as a function of $T$.

On the border of the superheated and saturated region, the vapour fraction and temperature profiles obey the gradient-continuity conditions (i.e. $T, c$, $\mathrm{d} T / \mathrm{d} y$ and $\mathrm{d} c / \mathrm{d} y$ are continuous at $y=\delta_{a}$ ). Combining these equations to eliminate $y$ yields $\mathrm{d} F / \mathrm{d} T$ $=\mathrm{d} G / \mathrm{d} T$, and on substituting eq. (10) we get

$$
\left.\frac{\mathrm{d} F}{\mathrm{~d} T}\right|_{z_{a}}=\left.\frac{\mathrm{d} G}{\mathrm{~d} T}\right|_{i_{a}}=\frac{c_{b}-c_{a}}{T_{b}-T_{a}} \frac{\delta_{t}-\delta_{a}}{\delta_{c}-\delta_{a}}
$$

Equation (16) prescribes mathematically that $\left(T_{a}, c_{a}\right)$ is situated on the saturation line in such a way that the straight line (10) of the superheated region is tangential to the saturation line $F(T)$ at $\left(T_{a}, c_{a}\right)$. Thus, $\left(T_{a}, c_{a}\right)$ coincide with $\left(T_{b}, c_{b}\right)$ when the bulk is satur- ated and $\delta_{i}=\delta_{c}$, implying that the entire film is saturated when the bulk is saturated. This feature of the film for small vapour mass fractions (or for $L e_{v}$ $=1$ ) is explained by Brouwers (1990). In Fig. 2 the determination of $\left(T_{a}, c_{a}\right)$ is represented graphically for the cases of a saturated and a superheated mixture.

The dimensionless fog layer thickness follows from the temperature-gradient-continuity condition, combined with eqs (8), (15), (16) and the first-order approximation of cq. (17) of Brouwcrs (1992) for small $\left|\varepsilon_{1}\right|$ and $\left|\varepsilon_{3}\right|$ :

$$
\frac{c_{b}-c_{a}}{c_{b}-c_{i}}=\frac{\delta_{c}-\delta_{a}}{\delta_{c}}
$$

as

$$
\frac{\delta_{a}}{\delta_{t}}=\frac{\operatorname{Le}\left(T_{a}-T_{i}\right)+\frac{H_{1 \mathrm{at}}}{c_{p}}\left(c_{\mathrm{a}}-c_{i}\right)}{\operatorname{Le}\left(T_{b}-T_{i}\right)+\frac{H_{\text {lat }}}{c_{p}}\left(c_{b}-c_{i}\right) \frac{\delta_{x}}{\delta_{c}}}
$$

Equation (17) is based on the assumption of an undisturbed (linear) vapour profile in the saturated part of the film; it is introduced to retain in the analysis the option of unequal thermal and diffusional film thickness. Some computations by Brouwers (1992) for some extreme examples of dilute water vapour in air mixtures indicated that the introduced error can be neglected in most practical situations.

The correction factor for the heat transferred is now obtained by comparing the heat transfer with fog formation with that without fog formation. Differentiating eq. (15) with respect to $y$, applying the saturation condition, and substituting eq. (18) results, according to the NIV fog film model, in the following thermal correction factor:

$$
\Theta_{r, f 1}=\frac{\left.\delta_{t} \frac{\mathrm{d} T}{\mathrm{~d} y}\right|_{y=0}}{\left(T_{b}-T_{i}\right)}=\frac{1+\frac{H_{\text {lat }}}{c_{p}} \frac{1}{L e} \frac{c_{b}-c_{i}}{T_{b}-T_{i}} \frac{\delta_{i}}{\delta_{c}}}{1+\left.\frac{H_{1 \mathrm{at}}}{c_{p}} \frac{1}{L e} \frac{\mathrm{d} F}{\mathrm{~d} T}\right|_{T_{i}}} .
$$

The NIV correction factor for mass transfer is obtained by the application of eqs (15), (18) and the saturation line:

$$
\Theta_{c, f 1}=\frac{\left.\delta_{c} \frac{\mathrm{d} c}{\mathrm{~d} y}\right|_{y=0}}{\left(c_{b}-c_{i}\right)}=\frac{1+\left(\frac{H_{\text {lat }}}{c_{p}} \frac{1}{L e} \frac{c_{b}-c_{i}}{T_{b}-T_{i}} \frac{\delta_{t}}{\delta_{c}}\right)^{-1}}{1+\left(\left.\frac{H_{\text {lat }}}{c_{p}} \frac{1}{L e} \frac{\mathrm{d} F}{\mathrm{~d} T}\right|_{T_{i}}\right)^{-1}} .
$$

The correction factors (19) and (20) are very compact and useful results, since they follow directly from the local bulk conditions, $\left(T_{b}, c_{b}\right)$, the interface conditions, $\left(T_{t}, c_{t}\right)$, and the saturation line, $F(T)$. To determine the correction factors the iterative calculation of $\left(T_{a}, c_{a}\right)$ and of the fog film thickness, $\delta_{a}$, are not necessary.

Moreover, the analytical expressions for the correction factors clarify the influence of the diverse para- 
meters and numerical results listed in Tables 1 and 3 of Brouwers (1992), concerning mixtures with dilute water vapour. When the slope condition (5) for condensation and eq. (6) for evaporation are considered [with eq. (4) inserted], one can readily see that $\Theta_{t, f 1}$ is larger (smaller) than unity for condensation (evaporation). A similar consideration of eq. (20), in combination with eqs (5) and (6), yields that $\Theta_{c, f 1}$ is smaller (larger) than unity for condensation (evaporation).

Furthermore, the thermal correction factor deviates more, and the diffusion correction factor less, from unity for larger $H_{1 a t} / c_{p} L e$. When it is borne in mind that $H_{1 a t} / c_{p} L e \equiv H_{1 a t} / c_{p, v} L e_{v}$, this feature of $\Theta_{t, f}$ and $\Theta_{c, f}$ is indeed confirmed by the results listed in the tables of Brouwers (1992). In Fig. 3, features of $\Theta_{t, f 1}$ and $\Theta_{c . s 1}$ are illustrated graphically for evaporation and condensation; $\tan p_{1}$ corresponds to $\mathrm{d} F / \mathrm{d} T$ in $T_{i}$ and $\tan p_{2}$ to $\left(c_{b}-c_{i}\right) /\left(T_{b}-T_{i}\right)$. Figure 3 also indicates that the correction factor differs most from unity when the bulk properties $\left(T_{b}, c_{b}\right)$ are situated on the saturation line $F(T)$, i.e. when the entire film is saturated.

Toor (1971a) derived analytically for $1-c_{i} \approx 1$, $L e=1$ and $\delta_{t}=\delta_{c}$ that the total (latent and sensible) heat transferred at the wall is not affected by fog formation. Applying the correction factors (19) and (20) one can verify that this feature of the film is also found when $L e \neq 1$ and $\delta_{t} \neq \delta_{c}$.

The amount of film fog is defined as

$$
\dot{m}_{f}=\int_{y=0}^{\delta_{a}} K \mathrm{~d} y=\frac{k}{H_{1 \mathrm{at}}}\left(\left.\frac{\mathrm{d} T}{\mathrm{~d} y}\right|_{y=0}-\left.\frac{\mathrm{d} T}{\mathrm{~d} y}\right|_{y-\delta_{a}}\right)
$$

[see eq. (13)].

The dimensionless amount of fog formed in the fog layer, defined as

$$
\dot{M}_{f}=\frac{\dot{m}_{f} H_{\text {lat }} \delta_{t}}{k\left(T_{b}-T_{i}\right)}
$$

follows from eqs (15), (18), (19), and (21), and the saturation condition

$$
\dot{M}_{f 1}=\Theta_{1, f 1}\left(1-\frac{1+\left.\frac{H_{\text {lat }}}{c_{p}} \frac{1}{L e} \frac{\mathrm{d} F}{\mathrm{~d} T}\right|_{T_{i}}}{1+\left.\frac{H_{\text {lat }}}{c_{p}} \frac{1}{L e} \frac{\mathrm{d} F}{\mathrm{~d} T}\right|_{T_{a}}}\right)
$$

This expression and eq. (19) indicate the augmentation of the NIV dimensionless fog formation with an increase in the difference between interface properties $\left(T_{i}, c_{i}\right)$ and boundary properties $\left(T_{a}, c_{a}\right)$, as well as when $H_{1 \mathrm{al}} / c_{p} L e$ is increased.

\section{APPLICATION OF THE NIV FOG FILM MODEL TO ChaNNGL. FLOW}

The application of the NIV fog model to the laminar or turbulent flow of a binary mixture through a channel is now discussed in detail. The governing equations for the vapour fraction and temperature alteration in the channel are derived for three possible cases: no fog formed; fog formed in the film but not in the bulk (since the mean mixed condition corresponds to superheat); and fog formed both in the film and in the bulk, which is saturated. Solutions in closed form will be provided for all three cases. It will be demonstrated that the applied NIV fog film model exhibits interesting features for Le equal to unity.

\section{Transfer of heat and mass without fog formation}

Consider the flow of a binary mixture through a channel of any given shape and in which there is a transfer of heat and mass from gas to a wall, and the vapour mass fraction is small, so that $\left|\varepsilon_{1}\right|$ is close to zero. When eqs (5) and (6) predict no formation of fog, the conventional film model is applicable. For small transfer rates the classical film model. correction factors, $\Theta_{z}$ and $\Theta_{c}$, tend to unity: for small $\left|\varepsilon_{1}\right|$ the zero-order approximation of the differential energy balance reads

$$
\frac{\mathrm{d} \bar{T}}{\mathrm{~d} x}=\frac{-4 h_{g}}{c_{p} D_{h}(\rho \bar{u})(x=0)}\left(\bar{T}-T_{i}\right)
$$

with the mean mixed temperature at the beginning of the channel as a boundary condition:

$$
\bar{T}(x=0)=T_{i n} \text {. }
$$

As the interface properties $\left(T_{i}, c_{i}\right)$ are assumed to be constant in the gas channel, eq. (24) can be integrated and the boundary condition (25) applied, yielding

$$
\bar{T}(x)=T_{i}+\left(T_{i n}-T_{i}\right) e^{\left[-4 h_{s} x / c_{p} D_{h}(\rho \bar{p})(x=0)\right]} .
$$

The first-order approximation for small $\left|\varepsilon_{1}\right|$ of the mass balance reads

$$
\frac{\mathrm{d} \bar{c}}{\mathrm{~d} x}=\frac{-4 g_{m}}{D_{h}(\rho \bar{u})(x=0)}\left(\bar{c}-c_{i}\right)
$$

with the bulk vapour mass fraction at the entrance of the channel as

$$
\bar{c}(x=0)=c_{i n} .
$$

Solving eq. (27) analytically with the application of boundary condition (28) leads to

$$
\bar{c}(x)=c_{i}+\left(c_{i n}-c_{i}\right) e^{\left[-4 g_{m} x / D_{h}(p \bar{u})(x=0)\right]} .
$$

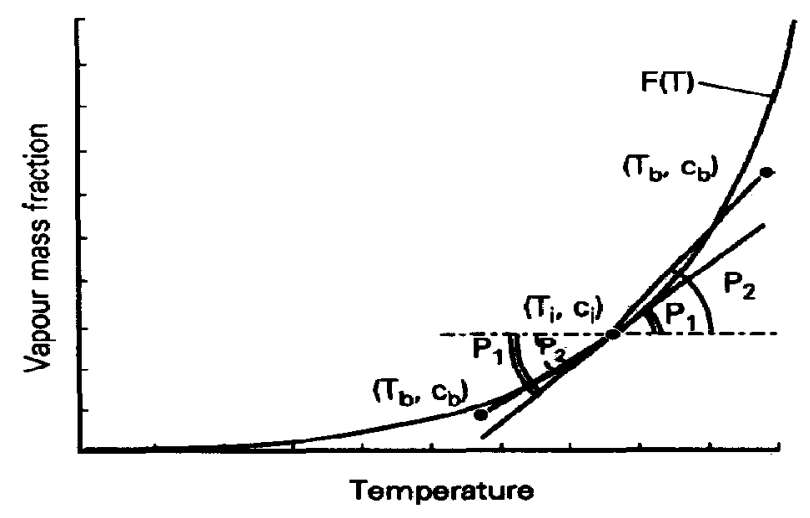

Fig. 3. Graphical representation of the effect of fog formation, $\delta_{z}=\delta_{c}$. 
Equations (26) and (29) give the bulk properties as a function of the coordinate in the direction of the flow, when the entire flow is superheated. These equations show that the mixed mean properties in the channel will lie between the interface values and entry values, as would be expected.

To determine the path of the bulk properties, the coordinate $x$ is eliminated from eqs (26) and (29), and $g_{m}=S h \rho \mathbb{D} / D_{h}$ and $h_{g}=N u k / D_{h}$ are substituted, yielding

$$
\bar{c}=\bar{G}(\bar{T})=c_{i}+\left(c_{i n}-c_{i}\right)\left(\frac{\bar{T}-T_{i}}{T_{i n}-T_{i}}\right)^{S h / N u L e}
$$

Equation (30) illustrates that for $L e$ equal to unity, and hence also the applicability of $N u=S h$, the mean mixed properties follow the relation between $c$ and $T$ in the superheated film represented by eq. (4). The classical, i.e. no fog, film model exhibits this feature as well, as was proved by Brouwers and Chesters (1992). So, the fact that for the special case of small $\left|\varepsilon_{1}\right|$ the same behaviour is found, is in fact to be expected.

Differentiating $\bar{G}(\bar{T})$ with respect to $\bar{T}$ reveals that $\bar{G}(\bar{T})$ is a monotonically increasing function of $\bar{T}$. For $S h / N u L e>1$ and condensation or $S h / N u L e<1$ and evaporation, $\bar{G}(\bar{T})$ proves to be a concave curve. For $S h / N u L e<1$ and condensation or $S h / N u L e>1$ and evaporation, $\bar{G}(\bar{T})$ is a convex curve. For $S h / N u$ Le $=1, \bar{G}(\bar{T})$ is a straight line, both for condensation or evaporation. These properties follow from the second derivative of $\bar{G}(\bar{T})$ with respect to $\bar{T}$.

As schematically depicted in Fig. 4, the bulk properties may move to intersect the saturation line, before mean mixed) situation may correspond to superheat. The differential energy balance then reads

$$
\frac{\mathrm{d} \bar{T}}{\mathrm{~d} x}=\Theta_{t, f 1} \frac{-4 h_{g}}{c_{p} D_{h}(\rho \bar{u})(x=0)}\left(\bar{T}-T_{i}\right)
$$

with boundary condition (25) and the differential vapour fraction balance

$$
\frac{\mathrm{d} \bar{c}}{\mathrm{~d} x}=\Theta_{c \cdot S 1} \frac{-4 g_{m}}{D_{h}(\rho \bar{u})(x=0)}\left(\bar{c}-c_{i}\right)
$$

with eq. (28) as the appropriate boundary condition.

The differential equations (31) and (32) are coupled because in the correction factors (19) and (20) $\bar{T}$ and $\bar{c}$ appear. Implicitly, the film model assumptions have thus been made in that $T_{b}$ and $c_{b}$ are taken to be $\bar{T}$ and $\bar{c}$, and that $\delta_{t}$ and $\delta_{c}$ are taken to be $D_{k} / N u$ and $D_{h} / S h$. Combining eqs (31) and (32) to eliminate $x$ and substitution of $h_{g}=k / \delta_{t}, g_{m}=\rho \mathbb{D} / \delta_{c}$, and eqs (19) and (20) yields

$$
\frac{\mathrm{d} \bar{c}}{\mathrm{~d} \bar{T}}=\frac{\mathrm{d} \bar{G}}{\mathrm{~d} \bar{T}}=\left.\frac{1}{L e} \frac{\mathrm{d} F}{\mathrm{~d} T}\right|_{T_{i}} .
$$

In order to express $\bar{c}$ as a function of $\bar{T}$, eq. (33) is integrated with respect to $\bar{T}$. and eqs (25) and (28) are applied, yielding

$$
\bar{c}=\bar{G}(\bar{T})=c_{i n}+\left.\left(\bar{T}-T_{i n}\right) \frac{1}{L e} \frac{\mathrm{d} F}{\mathrm{~d} T}\right|_{T_{i}} .
$$

Equation (34) is substituted into eq. (19) to eliminate $\bar{c}$, and the result is substituted into eq. (31). The variables $\bar{T}$ and $x$ in the resulting differential equation are separated and $\delta_{t} / \delta_{c}=S h / N u$ inserted, resulting in

$$
\frac{1+\left.\frac{H_{\text {lat }}}{c_{p}} \frac{1}{L e} \cdot \frac{\mathrm{d} F}{\mathrm{~d} T}\right|_{T_{i}}}{\bar{T}-T_{i}+\frac{H_{\text {lat }}}{c_{p}} \frac{S h}{L e N u}\left[c_{i n}-c_{i}+\left.\left(\bar{T}-T_{i n}\right) \frac{1}{L e} \frac{\mathrm{d} F}{\mathrm{~d} T}\right|_{T_{i}}\right]} \mathrm{d} \bar{T}=\frac{-4 h_{g}}{c_{p} D_{h}(\rho \bar{u})(x=0)} \mathrm{d} x .
$$

Integrating eq. (35) partially and applying boundary condition (25) yields

$$
\begin{gathered}
\ln \left\{\frac{\bar{T}-T_{i}+\frac{H_{\text {lat }}}{c_{p}} \frac{S h}{L e N u}\left[c_{i n}-c_{i}+\left.\left(\bar{T}-T_{i n}\right) \frac{1}{L e} \frac{\mathrm{d} F}{\mathrm{~d} T}\right|_{T_{i}}\right]}{\left.T_{i n}-T_{i}+\frac{H_{\text {lat }}}{c_{p}} \frac{S h}{L e N u} c_{i n}-c_{i}\right)}\right\} \\
=\frac{1+\left.\frac{H_{\text {lat }}}{c_{p}}\left(\frac{1}{L e}\right)^{2} \frac{S h}{N u} \frac{\mathrm{d} F}{\mathrm{~d} T}\right|_{T_{i}} \frac{-4 h_{g} x}{c_{p} D_{h}(\rho \bar{u})(x=0)}}{1+\left.\frac{H_{\text {lat }}}{c_{p}} \frac{1}{L e} \frac{\mathrm{d} F}{\mathrm{~d} T}\right|_{T_{i}}}
\end{gathered}
$$

which the film will become partially saturated. The governing equations of this physical situation are discussed below.

Heat and mass transfer with fog formation in the film but not in the bulk

When only a part of the film is saturated [as predicted by eq. (5) or (6)], the bulk (which is to say:
Equation (36) prescribes explicitly the temperature $\bar{T}(x)$ of the gas in the channel when fog is formed in the film and the bulk is still superheated. Substitution of $\bar{T}(x)$ according to eq. (36) into eq. (34) yields the local bulk vapour mass fraction in the channel.

Equation (33) indicates that, when fog is formed in a part of the flow and $L e=1$, the bulk properties shift in the direction of the saturation curve parallel to the 


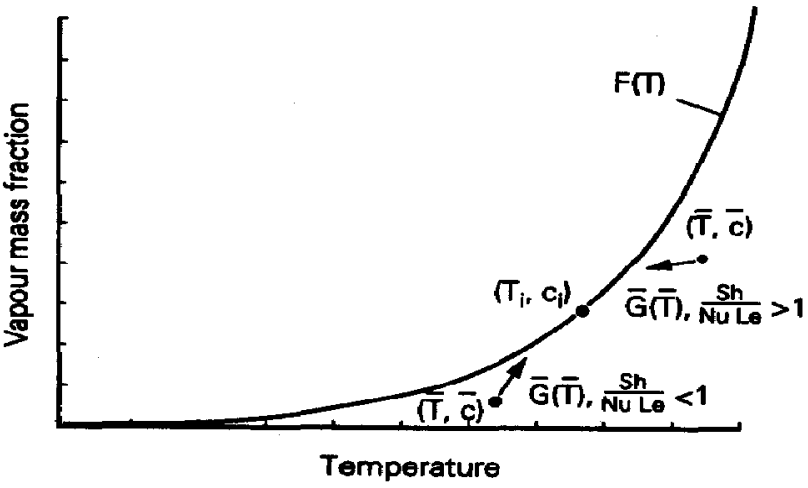

Fig. 4. Path of bulk properties in the case of no fog formation.

line tangent to $F(T)$ in $\left(T_{i}, c_{i}\right)$, as illustrated in Fig. 5. The shape of this path, which is a straight line, does not however depend on the magnitude of $S h / N u L e$.

The bulk properties follow this path until the saturation curve is reached. The behaviour of the mixture once the bulk is saturated is discussed below. When the heat and mass transfer in a channel are described without heeding the intersection of the saturation line and formation of fog, the bulk properties $(\bar{T}, \bar{c})$ may enter the supersaturated region following $\bar{G}(\bar{T})$. This phenomenon is described by eq. (30) and illustrated in Figs 4 and 5.

Heat and mass transfer with fog formation in both the film and the bulk

The last physical situation concerns a saturated bulk flow in which fog is formed. The entire film is then also saturated, thus $\left(T_{a}, c_{a}\right)$ coincide with $(\bar{T}, \bar{c})$. This property of the NIV analysis is mathematically prescribed by eq. (16) and is illustrated in Fig. 2. The conditions for bulk fog to be created in the gas flow are

$$
\frac{\mathrm{d} \bar{G}}{\mathrm{~d} \bar{T}}<\left.\frac{\mathrm{d} F}{\mathrm{~d} T}\right|_{\bar{T}}
$$

and

$$
\frac{\mathrm{d} \bar{G}}{\mathrm{~d} \bar{T}}>\left.\frac{\mathrm{d} F}{\mathrm{~d} T}\right|_{\bar{T}}
$$

for condensation and for evaporation, respectively [see Brouwers (1992), in which eq. (33) should be applied in the NIV case]. The bulk fog created keeps the bulk properties on the saturation line, $\bar{c}=F(\bar{T})$. The differential energy balance in the case of fogged bulk flow reads

$$
\frac{\mathrm{d} \bar{T}}{\mathrm{~d} x}=\frac{-4 h_{g}}{c_{p} D_{h}(\rho \bar{u})(x=0)}\left[\Theta_{t, f 1}\left(\bar{T}-T_{i}\right)-\frac{\overline{\dot{m}}_{f 1} H_{\text {lat }}}{h_{g}}\right]
$$

with the associated boundary condition (25). The differential vapour fraction balance is given as

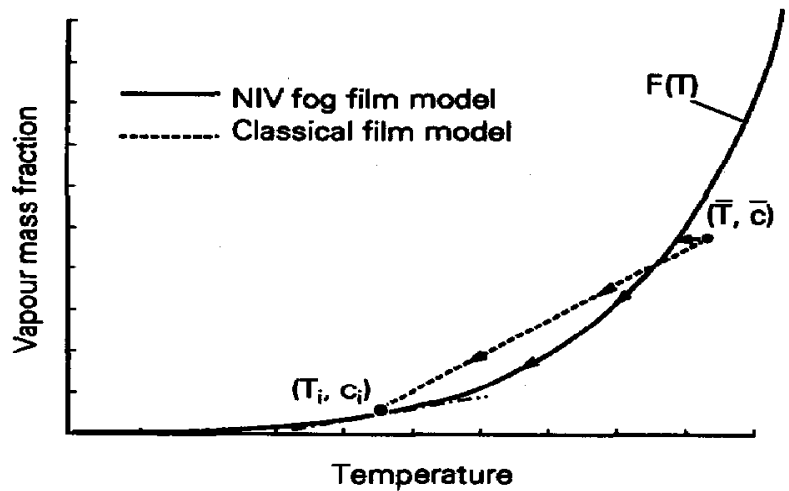

Fig. 5. Path of bulk properties in the case of wall condensation and fog formation, $L e=1$.

$$
\frac{\mathrm{d} \bar{c}}{\mathrm{~d} x}=\frac{-4 g_{m}}{D_{h}(\rho \bar{u})(x=0)}\left(\Theta_{c . S 1}\left(\bar{c}-c_{i}\right)+\frac{\bar{m}_{f 1}}{g_{m}}\right)
$$

with boundary condition (28). The quantity of fog $\overline{\dot{m}}_{f 1}$ is such that $\mathrm{d} \bar{G} / \mathrm{d} \bar{T}=\mathrm{d} F / \mathrm{d} T$ [eq. (55) from Brouwers (1992)] is fulfilled. This means mathematically that the mixture follows the saturation line while flowing through the channel. With eqs (19), (20), (39) and (40), $h_{g}=k / \delta_{t}, g_{m}=\rho \mathbb{D} / \delta_{c}$ and $\mathrm{d} \bar{c} / \mathrm{d} \bar{T} \equiv \mathrm{d} \bar{G} / \mathrm{d} \bar{T}=\mathrm{d} F / \mathrm{d} T$, the dimensionless quantity of fog in the bulk flow is obtained as

$$
\overline{\bar{M}}_{f 1}=\Theta_{t, f 1}\left(1-\frac{1+\left.\frac{H_{\text {lat }}}{c_{p}} \frac{1}{L e} \frac{\mathrm{d} F}{\mathrm{~d} T}\right|_{T_{t}}}{1+\left.\frac{H_{\mathrm{lat}}}{c_{p}} \frac{\mathrm{d} F}{\mathrm{~d} T}\right|_{T}}\right) .
$$

The thermal NIV correction factor figuring in this equation is found in eq. (19). Note that for $L e=1$ the amount of bulk fog, given by eq. (41), is identical to that created in the film, given by eq. (23).

The differential equations (39) and (40) are coupled because $\bar{c}=F(\bar{T})$. Hence, only $\bar{T}(x)$ has to be determined. Equation (41) is substituted into eq. (39):

$$
\begin{aligned}
\frac{\mathrm{d} \bar{T}}{\mathrm{~d} x}= & \Theta_{t, f 1} \frac{-4 h_{e}}{c_{p} D_{k}(\rho \bar{u})(x=0)}\left(\bar{T}-T_{i}\right) \\
& \times \frac{1+\left.\frac{H_{1 \mathrm{at}}}{c_{p}} \frac{1}{L e} \frac{\mathrm{d} F}{\mathrm{~d} T}\right|_{T_{i}}}{1+\left.\frac{H_{1 \mathrm{at}}}{c_{p}} \frac{\mathrm{d} F}{\mathrm{~d} T}\right|_{\bar{T}}} .
\end{aligned}
$$

By substitution of $\delta_{t} / \delta_{c}=S h / N u$ into eq. (19), substitution of the result into eq. (42), and separation of the variables $\bar{T}$ and $x$, one finds

$\frac{1+\left.\frac{H_{1 \mathrm{at}}}{c_{p}} \frac{\mathrm{d} F}{\mathrm{~d} T}\right|_{T}}{\bar{T}-T_{i}+\frac{H_{1 \mathrm{at}}}{c_{p}} \frac{S h}{N u \operatorname{Le}}\left(\bar{c}-c_{i}\right)} \mathrm{d} \bar{T}$

$$
=\frac{-4 h_{g}}{c_{p} D_{h}(\rho \bar{u})(x=0)} \mathrm{d} x .
$$


The left-hand side of this equation can be integrated analytically with respect to $\bar{T}$ when the product $S h / N u$ Le equals unity (which implies $L e=1$ for most practical cases since $S h / N u \approx L e^{p}$, where $0 \leqslant p<1$, e.g. according to the Chilton-Colburn analogy: $p=1 / 3$ ). Setting this product equal to unity, eq. (43) is partially integrated and the coupled boundary conditions (25) and (28) applied:

$$
\begin{aligned}
& \frac{\bar{T}(x)-T_{i}+\frac{H_{\text {lat }}}{c_{p}} \frac{1}{L e}\left[\bar{c}(x)-c_{i}\right]}{T_{\text {in }}-T_{i}+\frac{H_{\text {lat }}}{c_{p}} \frac{1}{L e}\left(c_{i n}-c_{i}\right)} \\
& =e^{\left[-4 h_{q} x / c_{p} D_{n}(\rho \bar{u})(x=0)\right]} .
\end{aligned}
$$

Equation (44) gives the temperature $\bar{T}(x)$ implicitly as a function of $x$, because $\bar{c}(x)=F[\bar{T}(x)]$. The bulk temperature and vapour mass fraction follow the saturation line in the direction of $\left(T_{i}, c_{i}\right)$ while flowing through the channel, see Fig. 5.

\section{RESULTS OF THE NIV FOG FILM MODEL AND INTRODUCTION OF THE COMPOUND FOG FILM MODEL}

The results of the fog model here derived will be calculated for the underlying conditions of Brouwers (1992) concerning various water-vapour air mixtures (note that $H_{1 a t} / c_{p} L e$ is identical to $H_{1 a t} / c_{p, v} L e_{v}$ ). On the basis of the here derived NIV fog correction factors and classical film model correction factors, compounded correction factors are introduced which account for both fog formation and the presence of an induced velocity. To determine fog formation, use has been made of the slope condition that reckons with the effect of the induced velocity: eqs (1) and (5).

The computed mass transfer correction factor, $\Theta_{c, r 1}$, listed in Table 1 corresponds to circumstances found in heat exchangers. It agrees extremely well with the ratio of the overall correction factor (taking account of both fog formation and the induced velocity) to the classical correction factor (taking account of an induced velocity, only), listed in Brouwers (1992) (referred to as: $\Theta_{c, f 1} / \Theta_{c}$ and $\Theta_{c, f 2} / \Theta_{c}$ ). This implies that the overall correction fator for fog formation and induced velocity can be approximated by multiplication of the correction factor of the NIV fog film model by the classical film model correction factor, i.e.

$$
\Theta_{c, f 2}=\Theta_{c} \Theta_{c, f 1} \text {. }
$$

Thus, the introduced correction factor $\Theta_{c .52}$ is compounded from the conventional correction factor $\Theta_{c}$ which accounts for suction or injection, and an independent NIV correction factor $\Theta_{c, f 1}$ which accounts for fog formation only.

In Table 2 the corresponding results are presented for the condenser situations. A comparison of the values in the tables again indicates the good agreement of the compounded correction factor with those of the more profound analysis. Even for the typical condenser conditions listed in Table 2, involving a significant induced velocity, the accuracy of the compounded correction factor is astonishingly good.

On the other hand, the thermal correction factor, $\Theta_{t, f 1}$, listed in Table 2, does not agree with the quotient of the thermal fog film model correction factor and the Ackermann correction factor, $\Theta_{t . f_{1}} / \Theta_{\text {t }}$

Table 1. Results of the NIV and compound fog film models for $\left(T_{i}, c_{i}\right)=(293 \mathrm{~K}$, 0.0144)

\begin{tabular}{lccccc}
\hline & $L e_{v}$ & $\Theta_{t, S 1}$ & $\Theta_{i, f 1} \Theta_{c} / \Theta_{t}$ & $\Theta_{c, s 1}$ & $\bar{M}_{f 2}$ \\
\hline$T_{b}=303 \mathrm{~K}, c_{b}=0.0264$ & 0.50 & 1.222 & 1.215 & 0.922 & 0.392 \\
& 0.75 & 1.192 & 1.189 & 0.899 & 0.351 \\
& 1.00 & 1.169 & 1.169 & 0.882 & 0.317 \\
$T_{b}=333 \mathrm{~K}, c_{b}=0.1318$ & 1.25 & 1.151 & 1.152 & 0.868 & 0.288 \\
& 0.50 & 2.538 & 2.387 & 0.781 & 2.065 \\
& 0.75 & 2.328 & 2.281 & 0.717 & 1.902 \\
& 1.00 & 2.169 & 2.169 & 0.667 & 1.753 \\
& 1.25 & 2.044 & 2.069 & 0.629 & 1.626 \\
\hline
\end{tabular}

Table 2. Results of the NIV and compound fog film models for $\left(T_{i}, c_{i}\right)=(367.81 \mathrm{~K}$, 0.75)

\begin{tabular}{lccccc}
\hline & $L e_{v}$ & $\Theta_{t, f 1}$ & $\Theta_{t, f 1} \Theta_{d} / \Theta_{t}$ & $\Theta_{c, f 1}$ & $\bar{M}_{f 2}$ \\
\hline$T_{b}=370.63 \mathrm{~K}, c_{b}=0.875$ & 0.80 & 1.097 & 1.017 & 0.998 & 0.025 \\
& 0.90 & 1.097 & 1.060 & 0.998 & 0.121 \\
& 1.00 & 1.097 & 1.097 & 0.998 & 0.198 \\
$T_{b}=372.90 \mathrm{~K}, c_{b}=0.995$ & 0.80 & 1.000 & 1.000 & 1.000 & 0.000 \\
& 0.90 & 1.189 & 1.077 & 0.997 & 0.345 \\
& 1.00 & 1.188 & 1.188 & 0.996 & 0.778 \\
& 1.10 & 1.188 & 1.295 & 0.996 & 1.133 \\
\hline
\end{tabular}


and $\Theta_{t, f 2} / \Theta_{t}$ of Brouwers (1992). In other words, multiplying the thermal NIV fog correction factor derived here by the Ackermann correction factor does not yield a satisfactory compounded thermal correction factor. In general, all thermal and diffusional correction factors of the fog film models obey the basic equation (27) of Brouwers (1992), and thus the model and the needed computational time. Furthermore, the forms of $\Theta_{c}, \Theta_{t, s_{1}}$ and $\Theta_{c, S_{1}}$ as such are far less complex than the expressions derived in Brouwers (1992), and hence are much faster to determine.

In Tables 1 and 2 the amount of bulk fog formed, according to the compound fog film model derived here, is listed. Calculating

$$
\bar{M}_{f 2}=\frac{\left.\Theta_{t, f 2} \frac{\mathrm{d} F}{\mathrm{~d} T}\right|_{\bar{T}}-\frac{S h}{N u} \Theta_{c, s 2} \frac{\bar{c}-c_{i}}{1-c_{i}}\left(\left.\frac{1}{L e_{v}} \frac{\mathrm{d} F}{\mathrm{~d} T}\right|_{\bar{T}}+\frac{1}{L e} \frac{1-\bar{c}}{\bar{T}-T_{i}}\right)}{\left.\frac{\mathrm{d} F}{\mathrm{~d} T}\right|_{\bar{T}}+(1-\bar{c}) \frac{L e_{v}}{L e} \frac{c_{p, v}}{H_{\mathrm{lat}}}}
$$

heuristically constructed correction factors $\Theta_{c, y_{2}}$ and $\Theta_{t, f_{2}}$ have to meet these requirements as well. The thermal correction factor $\Theta_{t, r 1}$ has therefore to be multiplied by the same factor as $\Theta_{c, f_{1}}$, that is to say $\Theta_{c}$ :

$$
\Theta_{t, f 2}=\Theta_{c} \Theta_{t, f 1}
$$

This thermal fog correction factor has been divided by the Ackermann correction factor, $\Theta_{i}$, and the result listed in Tables 1 and 2. These tables reveal that the constructed fog thermal correction factor (46) agrees well when it is compared with the results listed in the tables referred to. Moreover, extensive calculations have been carried out with numerous other physical conditions, $L e_{v}$ and $H_{1 \mathrm{av}} / c_{p, v}$. All calculations proved that the accuracy is of the same order as the examples treated here. This implies $\Theta_{c, f 2}$ and $\Theta_{t, f_{2}}$ are good approximations for the fog film model correction factors, accounting for both the influence of fog and the induced velocity.

The compound fog film model is applied in the same way to closed-channel flow as the fog film model. The procedure is explained by Brouwers (1992), and illustrated here by Fig. 6. The main difference is that, to determine $\Theta_{t, f 2}$ and $\Theta_{c, 52}$, the boundary properties $\left(T_{a}, c_{a}\right)$ do not have to be calculated iteratively. This essential simplification is a major advantage since it reduces the complexity of the fog [see Brouwers (1992)] to determine the dimensionless bulk fog, Le has again been set equal to unity and $\mathrm{Nu}$ $=S h$. The fog film model correction factors have been applied (instead of $\Theta_{t}$ and $\Theta_{c}$ ) as for all considered cases the films are fogging. The calculated rates agree well with the corresponding ones listed in Brouwers (1992), once more proving the reliability of the compound fog film model expressions.

\section{COMPARISON OF THE FOG FILM MODEL WITH CONVECTIVE MODELS AND EXPERIMENTS}

In this section, the non-convective fog film model is compared rigorously with comprehensive models and experiments of previous investigators. These two-dimensional models concern laminar free and forced convective heat and mass transfer of dilute watervapour air mixtures to a wall, the presence of fog being described by the saturation condition.

\section{Free convective heat and mass transfer}

Koch (1986) investigated the free convective boundary-layer flow along a vertical cryosurface with wall condensation. To this end the governing equations of continuity, momentum, energy, molecular diffusion, and particle diffusion were derived and solved numerically. In this thesis it was demonstrated that the thermophoresis of particles is a significant mode of mass transfer when the interface temperature is below

Table 3. Relation between $S h / N u$ and $L e$, according to Bottemanne (1971) $(L e=63 / 71)$ and Gebhart and Pera (1971) $(L e=5 / 7)$, for free convection resulting from buoyancy

\begin{tabular}{|c|c|c|c|c|}
\hline \multirow[b]{2}{*}{$\frac{\beta_{c}\left(c_{b}-c_{t}\right)}{\beta_{t}\left(T_{b}-T_{i}\right)}$} & \multicolumn{2}{|c|}{ Gebhart and Pera (1971) } & \multicolumn{2}{|c|}{ Bottemanne (1971) } \\
\hline & {$\left[=\stackrel{S h / N u}{\left.C^{\prime}(0) / \phi^{\prime}(0)\right]}\right.$} & $L e \log \left(\frac{S h}{N u}\right)$ & {$\left[\begin{array}{c}S h / N u \\
\left.=\omega^{\prime}(0) / \vartheta^{\prime}(0)\right]\end{array}\right.$} & $L e \log \left(\frac{S h}{N u}\right)$ \\
\hline-0.5 & 0.81794 & 0.60 & & \\
\hline 0.0 & & & 0.94065 & 0.51 \\
\hline 0.25 & & & 0.94084 & 0.51 \\
\hline $\begin{array}{l}0.5 \\
0.67\end{array}$ & 0.84201 & 0.51 & 0.94100 & 051 \\
\hline $\begin{array}{l}0.07 \\
1.0\end{array}$ & 0.84278 & 0.51 & 0.94100 & 0.31 \\
\hline 1.5 & & & 0.94116 & 0.51 \\
\hline 2.0 & 0.84253 & 0.51 & & \\
\hline 4.0 & & & 0.94131 & 0.51 \\
\hline$\infty$ & & & 0.94146 & 0.50 \\
\hline
\end{tabular}
effects of both thermal and mass diffusion 


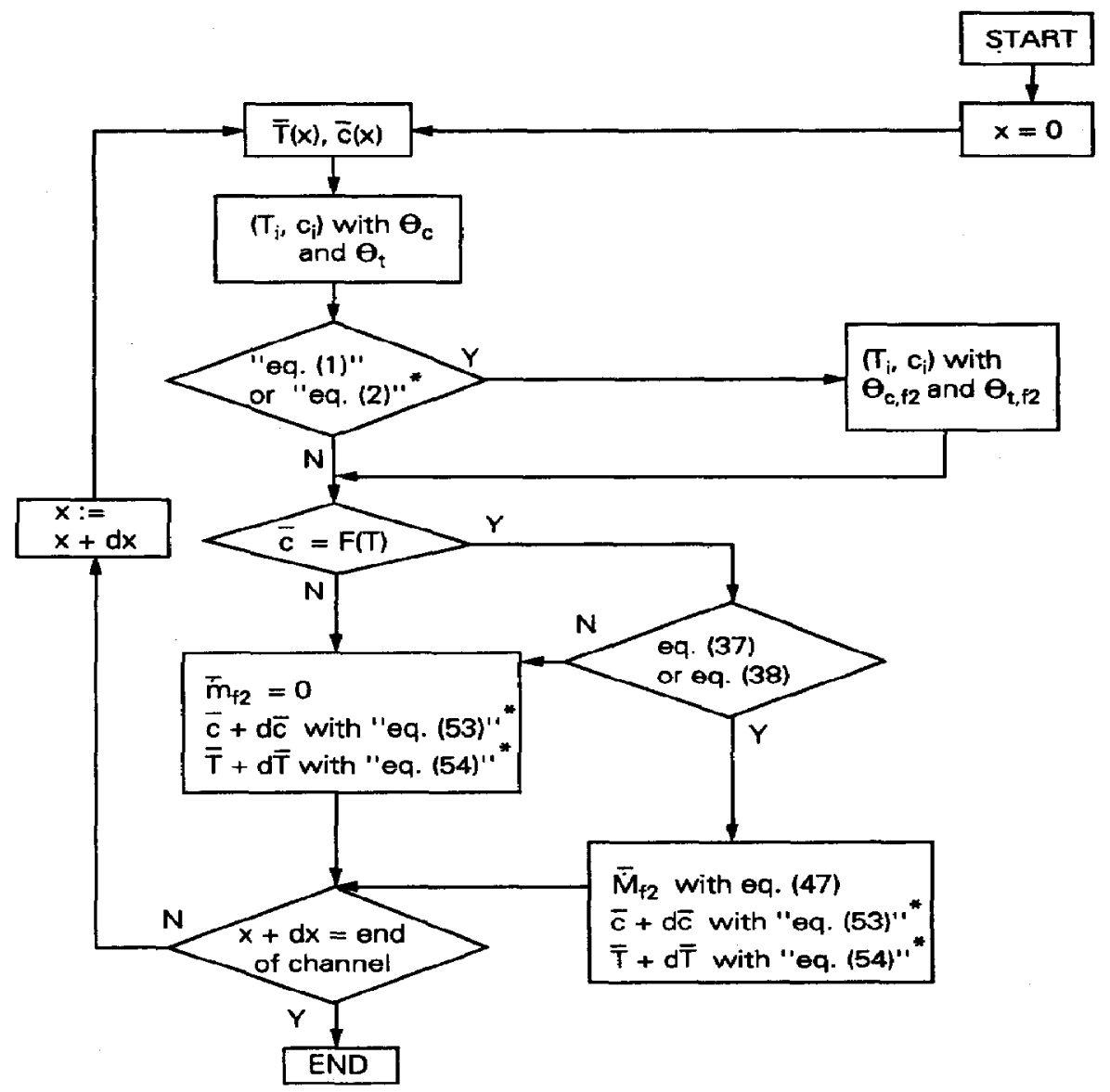

Fig. 6. Flow chart of the applied compound fog film model [*Brouwers (1992)].

Table 4. Free convective mass transfer of water-vapour air mixtures, $T_{b}=293 \mathrm{~K}\left[F\left(T_{b}\right)=0.0147\right]$, to a vertical wall, $T_{i}$ $=243 \mathrm{~K}$ and $c_{i}=F\left(T_{i}\right)=0.0003$

\begin{tabular}{|c|c|c|c|c|c|c|}
\hline$c_{b}$ & $\underset{\dot{m}_{\mathrm{hol}}\left(\mathrm{kg} \mathrm{m}^{-2} \mathrm{~s}^{-1}\right)}{\text { Experimental }}$ & $\begin{array}{c}\text { Theoretical } \\
\overline{\dot{m}}_{\text {tot }}\left(\mathrm{kg} \mathrm{m}^{-2} \mathrm{~s}^{-1}\right)\end{array}$ & $a_{c}$ & $\frac{\text { No fog }}{\bar{m}\left(\mathrm{~kg} \mathrm{~m}^{-2} \mathrm{~s}^{-1}\right)}$ & $\begin{array}{c}\text { Theoretical } \\
a_{c} \dot{\dot{m}}_{\text {tot }} / \overline{\dot{m}}\end{array}$ & $\begin{array}{c}\text { Eq. }(20) \\
\Theta_{c, J_{1}}\end{array}$ \\
\hline $\begin{array}{l}0.3 F\left(T_{b}\right) \\
0.6 F\left(T_{b}\right)\end{array}$ & $\begin{array}{l}1.08 \times 10^{-5} \\
1.72 \times 10^{-5}\end{array}$ & $\begin{array}{l}1.12 \times 10^{-5} \\
1.63 \times 10^{-5}\end{array}$ & $\begin{array}{l}0.63 \\
0.52\end{array}$ & $\begin{array}{l}1.51 \times 10^{-5} \\
3.16 \times 10^{-5}\end{array}$ & $\begin{array}{l}0.46 \\
0.26\end{array}$ & $\begin{array}{l}0.45 \\
0.26\end{array}$ \\
\hline
\end{tabular}

about $263 \mathrm{~K}$; it even dominates molecular mass transfer if $T_{i} \leqslant 213 \mathrm{~K}$. The theoretical model was furthermore found in good agreement with performed condensation experiments on a plate of $1 \mathrm{~m}$ height. In Table 4 two examples are listed, which will be compared with the predictions of the fog film model.

The total mean mass flux to the wall consists of two parts, namely mass transfer by particle and by molecular diffusion. As in this paper only the effect of fog formation on the (molecular) diffusion and energy equations is examined; in Table 4 also the purely molecular diffusion part, denoted as $a_{c}$, is included [taken from Fig. 5.20, Koch (1986)], as well as the diffusion mass transfer without fog formation. Dividing both diffusion mass transfer rates then pro- duces the correction factors for mass transfer. The diffusion correction factor obtained in this way can be compared with the predictions of the analysis presented here, since thermophoresis does not affect the coupled diffusion and energy equation.

Accordingly, $\Theta_{c, f 1}[$ see eq. (20)] is determined for the corresponding physical situations of Koch (1986): $L e=0.85, H_{1 a t} / c_{p}=2490 \mathrm{~K}$. For free convection flow over a vertical plate, originating from both temperature and concentration differences, the $S h / N u$ ratio can be approximated by

$$
\frac{\delta_{t}}{\delta_{c}}=\frac{S h}{N u}=L e^{0.51} .
$$

The computational results of Bottemanne (1971) 
$(L e \approx 0.89)$ and Gebhart and Pera $(1971)(L e \approx 0.71)$ confirm correlation (48) (see Table 3 ) since $0<\beta_{c}\left(c_{b}\right.$ $\left.-c_{\mathrm{f}}\right) / \beta_{t}\left(T_{b}-T_{i}\right)<0.12$ for all the cases examined in this section.

To compose the saturation line, the RankineKirchhoff equation of Koch (1986) is furthermore used for the water-vapour pressure: $\ln \left[\boldsymbol{P}_{v} /(\right.$ bar $\left.)\right]$ $=48.75-6825.7 T_{i} /(\mathrm{K})-5.144 \ln \left[T_{i} /(\mathrm{K})\right]$ (applicable for: $220 \mathrm{~K}<T_{i}<300 \mathrm{~K}$ ), and $P_{\text {tot }}=1$ bar. This liquid-water vapour pressure is employed, though the fog layer near the wall is below the solidification temperature of water. It was stated and employed by Koch (1986) that the droplets are in a metastable liquid state, and hence the mentioned saturation pressure of liquid water can be used. At the solid surface the saturation pressure of ice, of course, prevails, but the distinction with the water pressure is minor there and, moreover, $c_{i} \ll c_{b}$. In Table 4 the calculated $\Theta_{c, 51}$ for the two examined cases are listed.

The computed diffusion correction factors in Table 4 show that the fog film model mass transfer predictions are in good agreement; the values of $\Theta_{c, f 1}$ are predicted within a few per cent. One should realize that the results of Koch (1986) in Table 4 are based on the combined analysis of particle and molecular diffusion, and that $a_{\mathrm{c}}$ (the ratio of the molecular diffusion mass flux to the total mass flux at the surface) is read off graphically. In order to obtain data which can be compared more accurately with the fog film model, Koch and Straub (1990) utilized their numerical model to predict the free convective heat and mass transfer to a surface with $T_{i}=273 \mathrm{~K}, T_{b}=313 \mathrm{~K}$, and $c_{b}=0.3 F\left(T_{b}\right)$ or $c_{b}=0.6 F\left(T_{b}\right)$, thus excluding a relevant effect of thermophoresis.

For $c_{b}=0.3 F\left(T_{b}\right)$ the entire film is superheated; this follows from the slope condition (5), which was also pointed out by Koch and Straub (1990). For $c_{b}$ $=0.6 F\left(T_{b}\right)$, however, fog formation takes place in the mixture. Numerical calculations have been carried out by Koch and Straub (1990) with fog formation and thermophoresis suppressed, thermophoresis suppressed only, and with the complete model.

Their computations revealed that the solutions for thermophoresis included and suppressed are practically identical, implying that thermophoresis is indeed a negligible phenomenon for the interface and bulk conditions considered. Some determined heat fluxes to the wall are summarized in Table 5, while in Table 6 the mass fluxes are listed.

One can readily see that the transfer rates depend on the coordinate $x$, which constitutes the distance from the upper end of the vertical plate. The tables also indicate that the ratio of fog and no fog rates is nearly uniform, and thus independent of the level of heat and mass transfer. This aspect of the effect of fog formation is in qualitative accord with the film model approach, since the resulting correction factors only depend on interface and bulk conditions as well. The constant local transfer rates ratios furthermore imply that the total (and mean) heat and mass transfer fog/no fog ratios will have the same value as well. This
Table 5. Free convective heat transfer of water-vapour air mixtures, $T_{b}=313 \mathrm{~K}$ and $c_{b}=0.6 F\left(T_{b}\right)\left[F\left(T_{b}\right)=0.0471\right]$, to a vertical wall, $T_{i}=273 \mathrm{~K}$ and $c_{i}=0.00381$

\begin{tabular}{lcccc}
\hline$x(\mathrm{~m})$ & $\begin{array}{c}\text { No fog } \\
q\left(\mathbf{W ~ m}^{-2}\right)\end{array}$ & $\begin{array}{c}\text { Fog } \\
q\left(\mathbf{W ~ m}^{-2}\right)\end{array}$ & $\begin{array}{c}\text { Ratio } \\
\Theta_{t . f}\end{array}$ & $\begin{array}{c}\text { Eq. (19) } \\
\Theta_{t, f 1}\end{array}$ \\
\hline 0.05 & 213.0 & 300.9 & 1.41 & 1.46 \\
0.5 & 118.7 & 169.2 & 1.42 & 1.46 \\
1 & 99.70 & 142.5 & 1.43 & 1.46 \\
\hline
\end{tabular}

might be the reason why the overall agreement in Table 4 is so good.

In Tables 5 and 6 the NIV correction factors for fog formation are also included, in which $L e=0.86$ and $H_{\text {lat }} / c_{p}=2413 \mathrm{~K}$ have been substituted. The computed correction factors illustrate the reasonable agreement between the solution of the comprehensive free convective boundary layer equations, and those of the basic fog film model. The heat transfer ratios agree within $4 \%$, whereas the mass transfer ratios agree within $3 \%$.

\section{Forced convective heat and mass transfer}

Forced convective laminar flow between parallel plates (in the diffusional and thermal entrance region) with wall condensation of various dilute water vapours in air has been investigated theoretically and experimentally by Hayashi et al. (1981). The governing equations of continuity, diffusion and energy were derived, solved numerically and found in good agreement with performed experiments. On the basis of the saturation condition and a consideration of the vapour fraction and temperature profiles, Hayashi et al. (1981) derived the following condition for fog formation ( $p$ was referred to as $1 / T^{*}$ ):

$$
p=\frac{c_{b}-c_{i}}{\left.\left(T_{b}-T_{i}\right) \frac{\mathrm{d} F}{\mathrm{~d} T}\right|_{T_{i}}}>\frac{N u}{S h}
$$

which is identical to the film model condition for fog, eq. (5). It was furthermore derived that to the considered entrance region applies

$$
\frac{\delta_{c}}{\delta_{t}}=\frac{N u}{S h}=L e^{-0.36}=1.05
$$

as $L e=0.875$ for the studied mixtures. The theoretical and experimental results indicated that $\Theta_{\varepsilon, f}$ and $\Theta_{c, f}$ (referred to as $N u / N u_{0}$ and $S h / S h_{0}$, respectively) depend more on $p$ than on the local transfer rate (governed by the dimensionless distance from the entrance, referred to as $G z_{t}$ and $G z_{c}$ ). This observation is in qualitative accord with the principle of the fog film model.

In order to compare the results of Hayashi et al. (1981) quantitatively with the predictions of the fog film model, correction factors $\Theta_{t, f 1}$ and $\Theta_{c, f 1}$, represented by eqs (19) and (20), are determined for the process concerned. 
Table 6. Free convective mass transfer of water-vapour air mixtures, $T_{b}=313 . \mathrm{K}$ and $c_{b}=0.6 F\left(T_{b}\right)\left[F\left(T_{b}\right)=0.0471\right]$, to a vertical wall, $T_{i}=273 \mathrm{~K}$ and $c_{i}=0.00381$

\begin{tabular}{lcccc}
\hline$x(\mathrm{~m})$ & $\begin{array}{c}\text { No fog } \\
\dot{m}\left(\mathrm{~kg} \mathrm{~m}^{-2} \mathrm{~s}^{-1}\right)\end{array}$ & $\begin{array}{c}\text { Fog } \\
\dot{m}\left(\mathrm{~kg} \mathrm{~m}^{-2} \mathrm{~s}^{-1}\right)\end{array}$ & $\begin{array}{c}\text { Ratio } \\
\Theta_{c . f}\end{array}$ & $\begin{array}{c}\text { Eq. (20) } \\
\Theta_{c . f 1}\end{array}$ \\
\hline 0.05 & $1.391 \times 10^{-4}$ & $1.017 \times 10^{-4}$ & 0.73 & 0.71 \\
0.5 & $0.776 \times 10^{-4}$ & $0.559 \times 10^{-4}$ & 0.72 & 0.71 \\
1 & $0.652 \times 10^{-4}$ & $0.469 \times 10^{-4}$ & 0.72 & 0.71 \\
\hline
\end{tabular}

With eq. (49), eq. (19) is therefore rewritten as

$$
\Theta_{i, f 1}=\frac{1+\left.\frac{H_{\text {lat }}}{c_{p}} \frac{S h}{L e N u} \frac{\mathrm{d} F}{\mathrm{~d} T}\right|_{T_{i}} p}{1+\left.\frac{H_{\text {lat }}}{c_{p}} \frac{1}{L e} \frac{\mathrm{d} F}{\mathrm{~d} T}\right|_{T_{\mathrm{t}}}}
$$

and eq. (20) as

$$
\Theta_{c, f 1}=\Theta_{t, f 1} \frac{N u}{S h} \frac{1}{p}
$$

As relevant data in eqs (51) and (52) are now substituted by the aforesaid values of $L e$ and $N u / S h$, and $T_{i}=270 \mathrm{~K}, H_{1 a} / c_{p}=2500 \mathrm{~K}$. In eq. (51) the same water-vapour air saturation line is employed as in the previous free convective case. In Figs 7 and 8 the experimental data and theoretical results of Hayashi et al. (1981) (which were also based on $T_{i}=270 \mathrm{~K}$ ) are depicted, as are the computational results of eqs (51) and (52). These equations are used for $p \geqslant 1.05$, while $\Theta_{c, f 1}$ and $\Theta_{t, f 1}$ are unity for $p \leqslant 1.05$, since fog is not formed then.

Both figures illustrate the excellent agreement of the NIV fog film model with the theoretical and experimental results concerned. Some experimental results in Fig. 7, however, are typically situated above the theoretical predictions. These exceptions, though the deviations are modest, can be attributed to the variation in wall temperature during the experiments; $T_{i}$ ranged from 270 to $273 \mathrm{~K}$. Hence, eqs (51) and (52) are also evaluated with $T_{i}=273 \mathrm{~K}$ and all other values

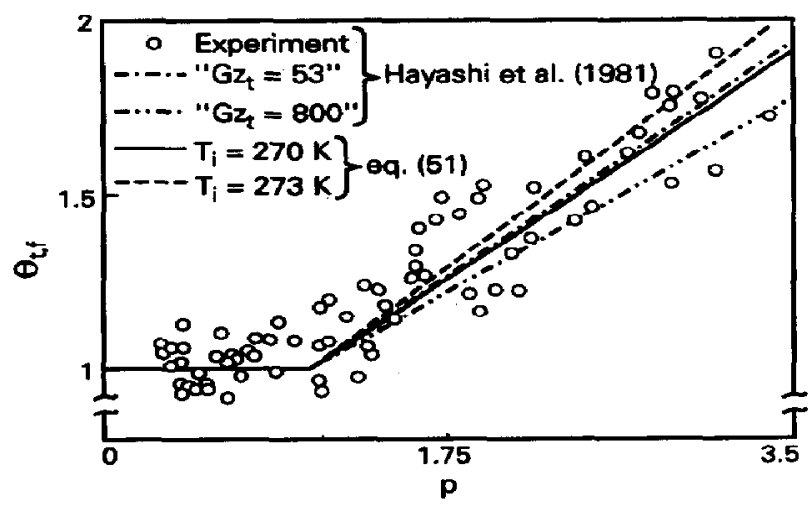

Fig. 7. Efiect of fog formation on forced convective heat transfer from a water-vapour air mixture to a wall in the presence of wall condensation. unchanged; the obtained results are also depicted in Figs 7 and 8. One can see in Fig. 8 that the effect of a higher $T_{i}$ is minor, while in Fig. 7 the line pertaining to $T_{i}=273 \mathrm{~K}$ has a steeper slope and is indeed in better agreement with some experimental data.

In this section the predictions of the NIV fog film model have been compared with those of convective models, and hence more comprehensive studies. This important comparison justifies the basic film model approach to fog formation, since it agrees with a deviation of typically $10 \%$. It has been shown and demonstrated by Brouwers and Chesters (1992) that the conventional film model allows for the induced velocity with a similar accuracy, which is well-acceptable for engineering end purposes. It is noteworthy that if the induced velocity were included in the analyses of Koch (1986), Koch and Straub (1990) and Hayashi et al. (1981), the effect of fog formation on transfer rates (and related correction factors) would be the same. In Section 4 we have seen that, both for fog and no fog formation, the heat and mass transfer is enhanced equally by the condensation-induced velocity.

\section{CONCLUSIONS}

In the present analysis, a film of a binary mixture has been examined in which there is a transfer of heat and mass. In this film, the induced velocity (or Stefan-Nusselt flow) has been assumed absent. The

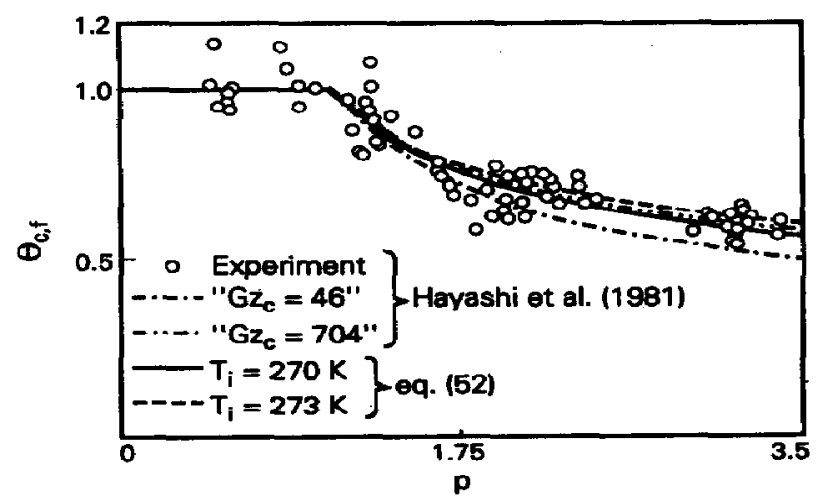

Fig. 8. Effect of fog formation on forced convective mass transfer from a water-vapour air mixture to a wall in the presence of wall condensation. 
error introduced by leaving this velocity out of consideration has been assessed thoroughly by means of an asymptotic analysis.

In the film with NIV, fog is allowed to form. First, a complete analysis has been given, on the basis of the saturation condition, of the presence and extent of a fog layer and a superheated layer. The governing equations of both regions have been derived, solved, and analytical expressions for the heat and mass transfer have been derived. On the basis of these expressions, compact NIV film model correction factors [eqs (19) and (20)] have been introduced, and the influence of the diverse parameters discussed.

Subsequently, the simple NIV fog film model correction factors have been applied to the flow of a mixture, with a small vapour mass fraction, through a channel. Three possible physical cases have been examined, namely a superheated flow without fog formation, a superheated bulk flow with fog formation in the film, and a saturated bulk flow with fog formation. For all three cases, solutions in closed form have been obtained, describing and illustrating the mixture's mean mixed temperature and vapour mass fraction behaviour with respect to the saturation line.

Calculation of the correction factors for realistic cases of fog formation in various air water-vapour mixtures indicated the significant effect of fog formation on heat and mass transfer. Furthermore, the NIV fog film model correction factors have been multiplied by the classical film model correction factors. These latter correction factors only allow for the effect of the induced velocity on heat and mass transfer, and not for the influence of fog formation. The compounded correction factors [eqs (45) and (46)] account for both formation of fog and the induced velocity. As these correction factors are sufficiently accurate and described by simple expressions, they are suited to and recommended for future engineering calculations.

The NIV fog film model correction factors have been compared with experimental and extensive theoretical studies of forced and free convective heat and mass transfer. Both studies concerned wall condensation of dilute water vapour in air and the effect of fog formation, with neglect of the induced velocity. The predictions of the basic NIV fog film model have been found to be in good accord with the results of the comprehensive studies considered.

Hence, the entire analysis yields the important conclusion that to compute free and forced convective systems with fog formation one can start from existing heat and mass transfer correlations (possibly multiplied by the classical film model correction factors for the effect of suction or injection). To investigate whether fog formation takes place, the film model provides the slope conditions (2) and (3) [which should be combined with eq. (1) to include also the case of an appreciable induced velocity]. If fog formation proves to be the case, the computed heat and mass transfer rates only need to be multiplied by correction factors (19) and (20), respectively, to include the effect of fog.
Acknowledgement-The author wants to thank the management of Akzo Research Laboratories Arnhem for their permission to publish this paper and Messrs. H. P. Korstanje and $G$. Vegt for their support of this work. He also wants to express his gratitude to Prof. A. K. Chesters of Eindhoven University of Technology for stimulating discussions on the subject.

\section{NOTATION}

$a_{c} \quad$ diffusional part of total mass flux at wall

$c \quad$ vapour mass fraction

$c_{p} \quad$ specific heat of mixture, $\mathrm{J} \mathrm{kg}^{-1} \mathrm{~K}^{-1}$

$c_{p, v} \quad$ specific heat of vapour, $\mathrm{J} \mathrm{kg}^{-1} \mathrm{~K}^{-1}$

Di diffusion coefficient, $\mathrm{m}^{2} \mathrm{~s}^{-1}$

$D_{h} \quad$ hydraulic diameter, four times the crosssectional/the perimeter, $m$

$F \quad$ saturation vapour mass fraction

$g_{m}$ mass transfer coefficient $\left(=S h \rho \mathbb{D} / D_{h}\right)$, $\mathrm{kg} \mathrm{m}^{-2} \mathrm{~s}^{-1}$

$G$ relation between vapour mass fraction and temperature in superheated region

$h_{g}$ heat transfer coefficient $\left(=N u k / D_{h}\right)$, $\mathbf{W} \mathbf{m}^{-2} \mathbf{K}^{-1}$

$H_{\text {lat }} \quad$ latent heat of condensation, $\mathrm{J} \mathrm{kg} \mathbf{~}^{-1}$

$k$ thermal conductivity, $W m^{-1} K^{-1}$

$K$ fog formation per unit volume, $\mathrm{kg} \mathrm{m}^{-3} \mathrm{~s}^{-1}$

Le Lewis number $\left(=k / \rho c_{p} \mathbb{D}\right)$

$L e_{v} \quad$ modified Lewis number $\left(=k / \rho c_{p, v} \mathbb{D}\right)$

$\dot{m} \quad$ mass flux at wall, $\mathrm{kg} \mathrm{m}^{-2} \mathrm{~s}^{-1}$

$\dot{m}_{f}$ fog mass flux in film defined in eq. (21), $\mathrm{kg} \mathrm{m}^{-2} \mathrm{~s}^{-1}$

$\dot{M}_{f}$ dimensionless fog formation defined in eq. (22)

$M_{n} \quad$ mass of one $\mathrm{kmol}$ of air, $\mathrm{kg}$

$M_{v} \quad$ mass of one kmol of water, $\mathrm{kg}$

$\mathrm{Nu} \quad$ Nusselt number

p

$\boldsymbol{P}$

dimensionless parameter defined locally

pressure, bar

heat flux at wall, $\mathrm{W} \mathrm{m}^{-2}$

Sh Sherwood number

$T$ temperature, $\mathrm{K}$

$u$ component of velocity in the $x$-direction, $\mathrm{m} \mathrm{s}^{-1}$

$x \quad$ coordinate along wall, $\mathrm{m}$

$y$ coordinate normal to wall, $m$

\section{Greek letters}

$\beta_{c} \quad$ volumetric coefficient of expansion with concentration $\left\{=\left(M_{n}-M_{v}\right) /\left[M_{v}+c_{b}\left(M_{n}\right.\right.\right.$ $\left.\left.\left.-M_{v}\right)\right]\right\}$

$\beta_{t} \quad$ volumetric coefficient of thermal expansion $\left(=1 / T_{b}\right), \mathrm{K}^{-1}$

$\delta \quad$ film thickness, $m$

$\varepsilon \quad$ perturbation quantity defined in eqs (2), (3) and (7)

$\Theta$ correction factor

$\rho$ density, $\mathrm{kg} \mathrm{m}^{-3}$

\section{Subscripts}

$a \quad$ border of saturated and superheated region b bulk

c diffusional 


$\begin{array}{ll}f & \text { fog } \\ f 1 & \text { pertaining to NIV fog film model } \\ f 2 & \text { pertaining to compound fog film model } \\ i & \text { interface } \\ \text { in } & \text { entry } \\ t & \text { thermal } \\ \text { tot } & \text { total } \\ & \\ \text { Superscripts } \\ -\quad \text { mean or "bulk" }\end{array}$

\section{REFERENCES}

Bottemanne, F. A., 1971, Theoretical solution of simultaneous heat and mass transfer by free convection about a vertical flat plate. Appl. Sci. Res. 25, 137-149.

Brouwers, H. J. H., 1990, Film models for transport phenomena with fog formation, with application to plastic heat exchangers and condensers. Ph.D. thesis, Eindhoven University of Technology, Eindhoven.

Brouwers, H. J. H., 1992, Film models for transport phenomena with fog formation: the fog film model. Int. $J$. Heat Mass Transfer 35, 13-25.

Brouwers, H. J. H. and Chesters, A. K., 1992, Film models for transport phenomena with fog formation: the classical film model. Int. J. Heat Mass Transfer 35, 1-11.

Dyke, M. van, 1975, Perturbation Methods in Fluid Mechanics. Parabolic Press, Stanford, CA.

Gebhart, B. and Pera, L., 1971, The nature of vertical natural convection flows resulting from the combined buoyancy effects of thermal and mass diffusion. Int. J. Heat Mass Transfer 14, 2025-2050.

Hayashi, Y., Takimoto, A. and Yamamoto, Y., 1981, Heat and mass transfer with mist formation in a laminar duct flow. Heat Transfer-Japan. Res. 10, 37-51.

Hills, A. W. D. and Szekely, J., 1964, Notes on vaporization into much colder surroundings. Chem. Engng Sci. 17, $79-81$.

Hills, A. W. D. and Szekely, J., 1969, A note on the enhancement of the diffusion limited vaporization rates by condensation within the thermal boundary layer. Int. $J$. Heat Mass Transfer 12, 111-114.

Koch, P., 1986, Wärme- und Stofftransport bei laminarer freier Konvektion in feuchter Luft an einer gekühlten vertikalen Platte. Ph.D. thesis, Technische Universität München, München (in German).

Koch, P. and Straub, J., 1990 (private communication).

Steinmeyer, D. E., 1972, Fog formation in partial condensers. Chem. Engng Prog. 68, 64-68.

Toor, H. L., $1971 \mathrm{a}$, Fog formation in boundary value problems. A.I.Ch.E. J. 17, 5-14.

Toor, H. L., 1971b, Fog vaporization and condensation in boundary value problems. Ind. Engng Fundam. 10, 121-131. 